\title{
ON THE CLASSIFICATION OF SIMPLE AMENABLE C*-ALGEBRAS WITH FINITE DECOMPOSITION RANK
}

\author{
GEORGE A. ELLIOTT AND ZHUANG NIU
}

Dedicated to Richard V. Kadison on the occasion of his ninetieth birthday

\begin{abstract}
Let $A$ be a unital simple separable $\mathrm{C}^{*}$-algebra satisfying the UCT. Assume that $\operatorname{dr}(A)<+\infty, A$ is Jiang-Su stable, and $\mathrm{K}_{0}(A) \otimes \mathbb{Q} \cong \mathbb{Q}$. Then $A$ is an ASH algebra (indeed, $A$ is a rationally $\mathrm{AH}$ algebra).
\end{abstract}

\section{INTRODUCTION}

Let $A$ be a simple separable nuclear unital $\mathrm{C}^{*}$-algebra. In [20, Matui and Sato showed that $A \otimes \mathrm{UHF}$ can be tracially approximated by finite dimensional $\mathrm{C}^{*}$-algebras (i.e., is $\mathrm{TAF}$ ) if $A$ is quasidiagonal with unique trace.

In this note, this result is enlarged upon as follows: the condition on the trace simplex is removed, at the cost of assuming the UCT, (still) finite nuclear dimension, and (still) that all traces are quasidiagonal - e.g., by assuming finite decomposition rank - see [2] — and (so far) of restricting the $\mathrm{K}_{0}$-group to have torsion-free rank equal to one.

Theorem 1.1. Let $A$ be a simple unital separable $C^{*}$-algebra satisfying the UCT. If $A \otimes Q$ has finite decomposition rank and $\mathrm{K}_{0}(A) \otimes \mathbb{Q} \cong \mathbb{Q}$, then $A \otimes Q \in \mathrm{TAI}$ (see Definition 2.11). In particular, $A \otimes \mathcal{Z}$ is classifiable, where $\mathcal{Z}$ is the Jiang-Su algebra (12]).

This theorem can also be regarded as an abstract version (still in a special case) of the classification result of [10] and [7, where any simple unital locally approximately subhomogeneous $\mathrm{C}^{*}$-algebra is shown to be rationally tracially approximated by Elliott-Thomsen algebras (1dimensional noncommutative CW complexes) ([7]) and hence to be classifiable ([10]).

\section{The MAIN RESUlt AND THE PROOF}

In this note let us use $Q$ to denote the UHF algebra with $\mathrm{K}_{0}(Q) \cong \mathbb{Q}$, and let us use tr to denote the canonical tracial state of $Q$.

Definition 2.1 (N. Brown, [3]). Let $A$ be a unital $\mathrm{C}^{*}$-algebra, and denote by $\mathrm{T}_{\mathrm{qd}}(A)$ the tracial states with the following property: For any $(\mathcal{F}, \varepsilon)$, there is a unital completely positive map $\phi: A \rightarrow Q$ such that

(1) $|\tau(a)-\operatorname{tr}(\phi(a))|<\varepsilon, a \in \mathcal{F}$, and

The research of the first author was supported by a Natural Sciences and Engineering Research Council of Canada (NSERC) Discovery Grant, and the research of the second author was supported by a Simons Foundation Collaboration Grant. 
(2) $\|\phi(a b)-\phi(a) \phi(b)\|<\varepsilon, a, b \in \mathcal{F}$.

Remark 2.2. In the original definition of a quasidiagonal trace (Definition 3.3.1 of [3]), the UHF algebra $Q$ was replaced by a matrix algebra. It is easy to see that these two approaches are equivalent.

Recall the tracial approximate uniqueness result of [6] and [16].

Theorem 2.3 (Theorem 4.15 of [6]; Theorem 5.3 of [16]). Let $A$ be a simple, unital, exact, separable $C^{*}$-algebra satisfying the $U C T$. For any finite subset $\mathcal{F} \subseteq A$ and any $\varepsilon>0$, there exist $n \in \mathbb{N}$ and $a \underline{\mathrm{K}}$-triple $(\mathcal{P}, \mathcal{G}, \delta)$ with the following property: For any admissible codomain $B$, and any three completely positive contractions $\phi, \psi, \xi: A \rightarrow B$ which are $\delta$-multiplicative on $\mathcal{G}$, with $\xi$ unital, $\phi$ and $\psi$ nuclear, and $\phi_{\#}(p)=\psi_{\#}(p)$ in $\underline{\mathrm{K}}(B)$ for all $p \in \mathcal{P}$, and such that $\phi(1)$ and $\psi(1)$ are unitarily equivalent projections, there exists a unitary $u \in \mathcal{U}_{n+1}(B)$ such that

$$
\left\|u^{*}\left(\begin{array}{cc}
\phi(a) & \\
& n \cdot \xi(a)
\end{array}\right) u-\left(\begin{array}{cc}
\psi(a) & \\
& n \cdot \xi(a)
\end{array}\right)\right\|<\varepsilon, \quad a \in \mathcal{F} .
$$

One may arrange that $u^{*}(\phi(1) \oplus n \cdot 1) u=\psi(1) \oplus n \cdot 1$.

Remark 2.4. In the theorem above, $n \cdot \xi(a)$ (or $n \cdot 1$ ) denotes the direct sum of $n$ copies of $\xi(a)$ (or 1). This notation is also used in the proof of Corollary 2.6 below.

Remark 2.5. In the theorem above (and also Corollary 2.6 below), one assumes by convention that the finite subset $\mathcal{G}$ is sufficiently large and $\delta$ is sufficiently small that $[\phi(p)]$ is well defined for any $p \in \mathcal{P}$ if a map $\phi$ is $\delta$-multiplicative on $\mathcal{G}$.

When $B=Q$, in fact one does not have to consider all the K-theory with coefficients. More precisely, one has

Corollary 2.6. Let $A$ be a simple, unital, exact, separable $C^{*}$-algebra satisfying the UCT. For any finite subset $\mathcal{F} \subseteq A$ and any $\varepsilon>0$, there exist $n \in \mathbb{N}$ and $a \underline{\mathrm{K}}$-triple $(\mathcal{P}, \mathcal{G}, \delta)$, with $\mathcal{P} \subseteq$ $\operatorname{Proj}_{\infty}(A)$, with the following property: For any three completely positive contractions $\phi, \psi, \xi$ : $A \rightarrow Q$ which are $\delta$-multiplicative on $\mathcal{G}$, with $\phi(1)=\psi(1)=1_{Q}-\xi(1)$ a projection, $[\phi(p)]_{0}=$ $[\psi(p)]_{0}$ in $\mathrm{K}_{0}(Q)$ for all $p \in \mathcal{P}$, and $\operatorname{tr}(\phi(1))=\operatorname{tr}(\psi(1))<1 / n$, where $\operatorname{tr}$ is the canonical tracial state of $Q$, there exists a unitary $u \in Q$ such that

$$
\left\|u^{*}(\phi(a) \oplus \xi(a)) u-\psi(a) \oplus \xi(a)\right\|<\varepsilon, \quad a \in \mathcal{F} .
$$

Proof. Applying Theorem 2.3 to $\mathcal{F}$ and $\varepsilon>0$, one obtains $n_{0} \in \mathbb{N}$ and a $\underline{\text { K-triple }}(\widetilde{\mathcal{P}}, \mathcal{G}, \delta)$ with the property of Theorem 2.3 . Set

$$
n_{0}+1=n \quad \text { and } \quad \widetilde{\mathcal{P}} \cap \operatorname{Proj}_{\infty}(A)=\mathcal{P} .
$$

Let us show that $n$ and $(\mathcal{P}, \mathcal{G}, \delta)$ have the desired property.

Let $\phi, \psi, \xi: A \rightarrow Q$ be completely positive contractions which are $\delta$-multiplicative on $\mathcal{G}$, with $\phi(1)=\psi(1)=1_{Q}-\xi(1)$ a projection, $[\phi(p)]_{0}=[\psi(p)]_{0}$ in $\mathrm{K}_{0}(Q)$ for all $p \in \mathcal{P}$, and $\operatorname{tr}(\phi(1))=\operatorname{tr}(\psi(1))<1 / n$. 
Decompose $\xi$ approximately on $\mathcal{F} \subseteq A$ as a repeated direct sum

$$
\underbrace{\xi^{\prime} \oplus \cdots \oplus \xi^{\prime}}_{n_{0}}
$$

where $\xi^{\prime}: A \rightarrow Q$ is again a completely positive contraction which is (necessarily, if the approximation is sufficiently good) $\delta$-multiplicative on $\mathcal{G}$, and $\left(\xi^{\prime} \oplus \cdots \oplus \xi^{\prime}\right)\left(1_{A}\right)=\xi\left(1_{A}\right)$. Since $\operatorname{tr}(\phi(1))=\operatorname{tr}(\psi(1))<1 / n$, one has that

$$
\operatorname{tr}(\xi(1))>(n-1) / n=n_{0} / n,
$$

and so

$$
\phi(1)=\psi(1) \preceq e,
$$

where $e=\xi^{\prime}(1)$. Then the maps $\phi \oplus \xi$ and $\psi \oplus \xi$ have the forms

$$
\phi \oplus\left(n \cdot \xi^{\prime}\right), \psi \oplus\left(n \cdot \xi^{\prime}\right): A \rightarrow \mathrm{M}_{n_{0}+1}(e Q e),
$$

respectively. Note that $e Q e$ is stably isomorphic to $Q$, and therefore

$$
\mathrm{K}_{0}(e Q e, \mathbb{Z} / k \mathbb{Z})=\{0\}, \quad k \in \mathbb{N} \backslash\{0\}, \quad \text { and } \quad \mathrm{K}_{1}(e Q e, \mathbb{Z} / k \mathbb{Z})=\{0\}, \quad k \in \mathbb{N} \cup\{0\} .
$$

Together with the assumption $[\phi(p)]_{0}=[\psi(p)]_{0}$ in $\mathrm{K}_{0}(Q)$ for all $p \in \mathcal{P}$, this implies

$$
\phi_{\#}(p)=\psi_{\#}(p) \in \underline{\mathrm{K}}(e Q e), \quad p \in \widetilde{\mathcal{P}} .
$$

Thus, it follows from Theorem 2.3 that there is a unitary $w \in \mathrm{M}_{n_{0}+1}(e Q e)$ such that

$$
\left\|w^{*}\left(\begin{array}{cc}
\phi(a) & \\
& n_{0} \cdot \xi^{\prime}(a)
\end{array}\right) w-\left(\begin{array}{cc}
\psi(a) & \\
& n_{0} \cdot \xi^{\prime}(a)
\end{array}\right)\right\|<\varepsilon, \quad a \in \mathcal{F},
$$

and

$$
w^{*}\left(\phi(1) \oplus n_{0} \cdot e\right) w=\psi(1) \oplus n_{0} \cdot e .
$$

Note that

$$
\phi(1) \oplus\left(n_{0} \cdot e\right)=\psi(1) \oplus\left(n_{0} \cdot e\right)=1_{Q} .
$$

By (2.1), a straightforward calculation shows that

$$
u:=1_{Q} w 1_{Q}
$$

is a unitary of $Q$. Clearly, if the approximation of $\xi$ by $\xi^{\prime} \oplus \cdots \oplus \xi^{\prime}$ on $\mathcal{F}$ is sufficiently good, then, in $Q$,

$$
\left\|u^{*}(\phi(a) \oplus \xi(a)) u-\psi(a) \oplus \xi(a)\right\|<\varepsilon, \quad a \in \mathcal{F}
$$

as desired.

Definition 2.7. Recall that an abelian group $G$ is said to be of (torsion free) rank one if $G \otimes \mathbb{Q} \cong \mathbb{Q}$. 
Lemma 2.8. Let $\Delta$ be a compact metrizable Choquet simplex. Then, for any finite subset $\mathcal{F} \subseteq$ $\operatorname{Aff}(\Delta)$ and any $\varepsilon>0$, there exist $m \in \mathbb{N}$ and unital (pointwise) positive linear maps $\varrho$ and $\theta$,

$$
\operatorname{Aff}(\Delta) \stackrel{\varrho}{\longrightarrow} \mathbb{R}^{m} \stackrel{\theta}{\longrightarrow} \operatorname{Aff}(\Delta),
$$

where the unit of $\mathbb{R}^{m}$ is $(1, \ldots, 1)$, such that

$$
\|\theta(\varrho(f))-f\|_{\infty}<\varepsilon, \quad f \in \mathcal{F} .
$$

Proof. By Theorem 5.2 of [14] and its corollary, there is an increasing sequence of finite-dimensional subspaces of $\operatorname{Aff}(\Delta)$ with dense union, containing the canonical order unit $1 \in \operatorname{Aff}(\Delta)$, and such that each map $\mathbb{R}^{m_{k}} \rightarrow \mathbb{R}^{m_{k+1}}$ and $\mathbb{R}^{m_{k}} \hookrightarrow \operatorname{Aff}(\Delta)$ is positive, with respect to the canonical (pointwise) order relations:

$$
\mathbb{R}^{m_{1} \hookrightarrow \longrightarrow} \mathbb{R}^{m_{2} \hookrightarrow \longrightarrow} \cdots \hookrightarrow \operatorname{Aff}(\Delta)
$$

(The authors are indebted to David Handelman for reminding us of [14].)

Without loss of generality, one may assume that $\mathcal{F} \subseteq \mathbb{R}^{m_{1}}$, and hence one only has to extend the identity map of $\mathbb{R}^{m_{1}}$ to a positive unital map $\varrho: \operatorname{Aff}(\Delta) \rightarrow \mathbb{R}^{m_{1}}$.

Write $\mathbb{R}^{m_{1}}=\mathbb{R} e_{1} \oplus \mathbb{R} e_{2} \oplus \cdots \oplus \mathbb{R} e_{m_{1}}$, and consider the unital positive functionals

$$
\rho_{i}: \mathbb{R}^{m_{1}} \ni\left(x_{1}, x_{2}, \ldots, x_{m_{1}}\right) \mapsto x_{i} \in \mathbb{R}, \quad i=1, \ldots, m_{1} .
$$

By the Riesz Extension Theorem ([21]), each $\rho_{i}$ can be extended to a unital positive linear functional $\tilde{\rho}_{i}: \operatorname{Aff}(\Delta) \rightarrow \mathbb{R}$. Then the map

$$
\varrho: \operatorname{Aff}(\Delta) \ni f \mapsto\left(\tilde{\rho}_{1}(f), \tilde{\rho}_{2}(f), \ldots, \tilde{\rho}_{m_{1}}(f)\right) \in \mathbb{R}^{m_{1}}
$$

has the desired property.

Lemma 2.9. Let $C=\underset{\lim }{\longrightarrow}\left(C_{n}, \iota_{n}\right)$ be a unital inductive system of $C^{*}$-algebras such that $C$ is simple. Let $\left(\mathbb{R}^{m},\|\cdot\|_{\infty}, \overrightarrow{u)}\right.$ be a finite-dimensional ordered Banach space with order unit $u$, and let $\gamma: \mathbb{R}^{m} \rightarrow \operatorname{Aff}\left(\mathrm{T}(C)\right.$ ) be a unital positive linear map. Then, for any finite set $\mathcal{F} \subseteq \mathbb{R}^{m}$ and any $\varepsilon>0$, there are $n$ and a unital positive linear map $\gamma_{n}: \mathbb{R}^{m} \rightarrow \operatorname{Aff}\left(\mathrm{T}\left(C_{n}\right)\right)$ such that

$$
\left\|\gamma(a)-\iota_{n, \infty} \circ \gamma_{n}(a)\right\|<\varepsilon, \quad a \in \mathcal{F}
$$

Proof. Denote by $e_{i}, i=1, \ldots, m$, the standard basis of $\mathbb{R}^{m}$, and write

$$
u=c_{1} e_{1}+\cdots+c_{m} e_{m}
$$

where $c_{1}, \ldots, c_{m}>0$. Since $C$ is simple, each affine function $\gamma\left(e_{i}\right)$ is strictly positive on $\mathrm{T}(C)$. Since $\mathrm{T}(C)$ is compact, there is $\delta_{i}$ such that

$$
\gamma\left(e_{i}\right)(\tau)>\delta_{i}, \quad \tau \in \mathrm{T}(C), 1 \leq i \leq m
$$

Without loss of generality, one may assume that $\mathcal{F}=\left\{e_{1}, e_{2}, \ldots, e_{m}\right\}$.

Pick $C_{n}$ and $e_{1}^{\prime}, e_{2}^{\prime}, \ldots, e_{m-1}^{\prime} \in \operatorname{Aff}\left(\mathrm{T}\left(C_{n}\right)\right)$ such that

$$
\left\|\iota_{n, \infty}\left(e_{i}^{\prime}\right)-\gamma\left(e_{i}\right)\right\|_{\infty}<\min \left\{\varepsilon, \frac{\delta_{i}}{2}, \frac{c_{m} \delta_{m}}{2\left(c_{1}+\cdots+c_{m-1}\right)}, \frac{c_{m} \varepsilon}{2\left(c_{1}+\cdots+c_{m-1}\right)}\right\}, \quad 1 \leq i \leq m-1 .
$$


In particular, by (2.2),

$$
\iota_{n, \infty}\left(e_{i}^{\prime}\right)(\tau) \geq \delta_{i} / 2, \quad \tau \in \mathrm{T}(C), 1 \leq i \leq m-1 .
$$

Setting

$$
e_{m}^{\prime}:=\frac{1}{c_{m}}\left(1-c_{1} e_{1}^{\prime}-\cdots-c_{m-1} e_{m-1}^{\prime}\right) \in \operatorname{Aff}\left(\mathrm{T}\left(C_{n}\right)\right)
$$

one has

$$
\begin{aligned}
\left\|\iota_{n, \infty}\left(e_{m}^{\prime}\right)-\gamma\left(e_{m}\right)\right\|_{\infty}= & \| \frac{1}{c_{m}}\left(1-c_{1} \iota_{n, \infty}\left(e_{1}^{\prime}\right)-\cdots-c_{m-1} \iota_{n, \infty}\left(e_{m-1}^{\prime}\right)\right)- \\
& \frac{1}{c_{m}}\left(1-c_{1} \gamma\left(e_{1}\right)-\cdots-c_{m-1} \gamma\left(e_{m-1}\right)\right) \|_{\infty} \\
\leq & \min \left\{\delta_{m} / 2, \varepsilon\right\} .
\end{aligned}
$$

In particular, by (2.2),

$$
\iota_{n, \infty}\left(e_{m}^{\prime}\right)(\tau) \geq \delta_{m} / 2, \quad \tau \in \mathrm{T}(C) .
$$

Then, considering instead the images of $e_{1}^{\prime}, e_{2}^{\prime}, \ldots, e_{m}^{\prime}$ in a building block further out (replacing $n$ by the later index), one may assume that

$$
e_{i}^{\prime}(\tau)>\delta_{i} / 4, \quad \tau \in \mathrm{T}\left(C_{n}\right), 1 \leq i \leq m
$$

In particular, all the affine functions $e_{i}^{\prime} \in \operatorname{Aff}\left(\mathrm{T}\left(C_{n}\right)\right)$ are positive. Define $\gamma_{n}: \mathbb{R}^{m} \rightarrow \operatorname{Aff}\left(\mathrm{T}\left(C_{n_{0}}\right)\right)$ by

$$
\gamma_{n}\left(e_{i}\right)=e_{i}^{\prime}, \quad 1 \leq i \leq m
$$

It is clear that $\gamma_{n}$ satisfies the condition of the lemma.

Theorem 2.10. Let $A$ be a separable simple unital exact $C^{*}$-algebra satisfying the UCT. Assume that $\mathrm{T}(A)=\mathrm{T}_{\mathrm{qd}}(A)$ and that $\mathrm{K}_{0}(A)$ is of rank one. Then, for any finite set $\mathcal{F} \subseteq A \otimes Q$ and any $\varepsilon>0$, there are unital completely positive linear maps $\phi: A \otimes Q \rightarrow I$ and $\psi: I \rightarrow A \otimes Q$, where $I$ is an interval algebra, such that

(1) $\phi$ is $\mathcal{F}$ - $\delta$-multiplicative, $\psi$ is an embedding, and

(2) $|\tau(\psi \circ \phi(a)-a)|<\varepsilon, a \in \mathcal{F}, \tau \in \mathrm{T}(A \otimes Q)$.

Proof. If $\mathrm{T}(A)=\varnothing$, then the conclusion holds trivially (with $I=\{0\}$ ). Otherwise, assuming, as we may, that $A \cong A \otimes Q$, we have $\mathrm{K}_{0}(A) \cong \mathbb{Q}$ (as order-unit groups).

Apply Corollary 2.6 to $A$ with respect to $(\mathcal{F} \cdot \mathcal{F}, \varepsilon / 4)$ to obtain $n$ and $(\mathcal{P}, \mathcal{G}, \delta)$. Since $\mathrm{K}_{0}(A)=\mathbb{Q}$ (unique unital identification), we may suppose that $\mathcal{P}=\left\{1_{A}\right\}$.

By Theorem 3.9 of [23], there is a simple unital inductive limit $C=\underline{\lim }\left(C_{i}, \iota_{i}\right)$ such that $\mathrm{K}_{0}(C)=\mathbb{Q}$ (unital identification), $C_{i}=\mathrm{M}_{k_{i}}(\mathrm{C}([0,1]))$, the maps $\iota_{i}$ are injective, and there is an isomorphism

$$
\Xi: \operatorname{Aff}(\mathrm{T}(A)) \cong \operatorname{Aff}(\mathrm{T}(C))
$$

By Lemma 2.8, there is an approximate factorization, by means of unital positive maps,

$$
\operatorname{Aff}(\mathrm{T}(A)) \stackrel{\varrho}{\longrightarrow} \mathbb{R}^{m} \stackrel{\theta}{\longrightarrow} \operatorname{Aff}(\mathrm{T}(A))
$$


such that

$$
\|\theta(\varrho(\hat{f}))-\hat{f}\|_{\infty}<\varepsilon / 16, \quad f \in \mathcal{F} .
$$

Therefore, by Lemma 2.9. after discarding finitely many terms of the sequence $\left(C_{i}, \iota_{i}\right)$, there is a unital positive linear map

$$
\gamma: \operatorname{Aff}(\mathrm{T}(A)) \stackrel{\varrho}{\longrightarrow} \mathbb{R}^{m} \longrightarrow \operatorname{Aff}\left(\mathrm{T}\left(C_{1}\right)\right)
$$

such that

$$
\left\|\left(\iota_{1, \infty}\right)_{*}(\gamma(\hat{f}))-\Xi(\hat{f})\right\|_{\infty}<\varepsilon / 8, \quad f \in \mathcal{F} .
$$

Denote by $\gamma^{*}: \mathrm{T}\left(C_{1}\right) \rightarrow \mathrm{T}(A)$ the affine map induced by $\gamma$ on tracial simplices. Since $\gamma$ factors through $\mathbb{R}^{m}$ (so that $\gamma^{*}$ factors through a finite dimensional simplex), there are $\tau_{1}, \ldots, \tau_{m} \in \mathrm{T}(A)$ and continuous functions $c_{1}, c_{2}, \ldots, c_{m}:[0,1] \rightarrow[0,1]$ such that

$$
\gamma^{*}\left(\tau_{t}\right)=c_{1}(t) \tau_{1}+c_{2}(t) \tau_{2}+\cdots+c_{m}(t) \tau_{m}, \quad t \in[0,1],
$$

and

$$
c_{1}(t)+c_{2}(t)+\cdots+c_{m}(t)=1, \quad t \in[0,1],
$$

where $\tau_{t} \in \mathrm{T}\left(C_{1}\right)$ is determined by the Dirac measure concentrated at $t \in[0,1]$.

Since $\tau_{1}, \tau_{2}, \ldots, \tau_{m} \in \mathrm{T}_{\mathrm{qd}}(A)$, there are unital completely positive linear maps $\phi_{k}: A \rightarrow Q$, $k=1,2, \ldots, m$, such that each $\phi_{k}$ is $\mathcal{G}$ - $\delta$-multiplicative, and

$$
\left|\operatorname{tr}\left(\phi_{k}(f)\right)-\tau_{k}(f)\right|<\varepsilon / 16 m, \quad f \in \mathcal{F} .
$$

For each $t \in[0,1]$, there is a open neighbourhood $U$ such that for any $s \in U$, one has

$$
\left|c_{k}(s)-c_{k}(t)\right|<1 / 4 m n .
$$

(Recall that $n$ is the constant from Corollary 2.6, as in the second paragraph of the proof.) Since $[0,1]$ is compact, there is a partition $0=t_{0}<t_{1}<\cdots<t_{l-1}<t_{l}=1$ such that

$$
\left|c_{k}(s)-c_{k}\left(t_{j}\right)\right|<1 / 4 m n, \quad s \in\left[t_{j-1}, t_{j}\right] .
$$

Moreover, we may assume that this partition is fine enough that

$$
\left|\gamma(\hat{f})\left(\tau_{t}\right)-\gamma(\hat{f})\left(\tau_{t_{j}}\right)\right|<\varepsilon / 8, \quad f \in \mathcal{F}, t \in\left[t_{j-1}, t_{j}\right] .
$$

For each $j=0,1, \ldots, l$, pick rational numbers $r_{j, 1}, r_{j, 2}, \ldots, r_{j, m} \in[0,1]$ such that

$$
r_{j, 1}+\cdots+r_{j, m}=1
$$

and

$$
\left|r_{j, k}-c_{k}\left(t_{j}\right)\right|<\min \{\varepsilon / 16 m, 1 / 4 m n\}, \quad k=1, \ldots, m .
$$

Write $r_{j, k}=q_{j, k} / p$ where $q_{j, k}, p \in \mathbb{N}$, and then define

$$
\varphi_{j}:=(\underbrace{\phi_{1} \oplus \cdots \oplus \phi_{1}}_{q_{j, 1}}) \oplus \cdots \oplus(\underbrace{\phi_{m} \oplus \cdots \oplus \phi_{m}}_{q_{j, m}}): A \rightarrow Q .
$$

Note that it follows from (2.4), (2.5), and (2.8) that

$$
\left|\operatorname{tr}\left(\varphi_{j}(f)\right)-\gamma^{*}\left(\tau_{t_{j}}\right)(f)\right|<\varepsilon / 4, \quad f \in \mathcal{F} .
$$


By (2.8), (2.6), one has that

$$
\frac{\left|q_{j, k}-q_{j+1, k}\right|}{p}<\frac{1}{m n}, \quad k=1, \ldots, m, j=0, \ldots, l-1 .
$$

For each $j=0, \ldots, l-1$, compare the direct sum maps

$$
\varphi_{j}=(\underbrace{\phi_{1} \oplus \cdots \oplus \phi_{1}}_{q_{j, 1}}) \oplus \cdots \oplus(\underbrace{\phi_{m} \oplus \cdots \oplus \phi_{m}}_{q_{j, m}})
$$

and

$$
\varphi_{j+1}=(\underbrace{\phi_{1} \oplus \cdots \oplus \phi_{1}}_{q_{j+1,1}}) \oplus \cdots \oplus(\underbrace{\phi_{m} \oplus \cdots \oplus \phi_{m}}_{q_{j+1, m}})
$$

and consider the common direct summand of these two maps,

$$
\psi_{j}:=(\underbrace{\phi_{1} \oplus \cdots \oplus \phi_{1}}_{\min \left\{q_{j, 1}, q_{j+1,1}\right\}}) \oplus \cdots \oplus(\underbrace{\phi_{m} \oplus \cdots \oplus \phi_{m}}_{\min \left\{q_{j, m}, q_{j+1, m}\right\}}) .
$$

By (2.10), one has

$$
\left|\operatorname{tr}\left(1-\psi_{j}(1)\right)\right|=\frac{1}{p} \sum_{k=1}^{m}\left|q_{j, k}-q_{j+1, k}\right|<\frac{1}{n} .
$$

On the other hand, since $\varphi_{j}$ and $\varphi_{j+1}$ are unital, one has

$$
\left[\left(\varphi_{j} \ominus \psi_{j}\right)\left(1_{A}\right)\right]_{0}=1-\operatorname{tr}\left(\psi_{j}\left(1_{A}\right)\right)=\left[\left(\varphi_{j+1} \ominus \psi_{j}\right)\left(1_{A}\right)\right]_{0} .
$$

Recall that $\mathcal{P}=\left\{1_{A}\right\}$. By the conclusion of Corollary 2.6 there is a unitary $u_{j+1}$ such that

$$
\left\|\varphi_{j}(f)-u_{j+1}^{*} \varphi_{j+1}(f) u_{j+1}\right\|<\varepsilon / 4, \quad f \in \mathcal{F} \cdot \mathcal{F}, 0 \leq j \leq l-1 .
$$

Define $v_{0}=1$, and set

$$
u_{j} u_{j-1} \cdots u_{1}=v_{j}, \quad j=1, \ldots, l .
$$

Then, for any $0 \leq j \leq l-1$ and any $f \in \mathcal{F} \cdot \mathcal{F}$, one has

$$
\begin{aligned}
& \left\|\operatorname{Ad}\left(v_{j}\right) \circ \varphi_{i}(f)-\operatorname{Ad}\left(v_{j+1}\right) \circ \varphi_{j+1}(f)\right\| \\
= & \left\|\left(u_{j} \cdots u_{1}\right)^{*} \varphi_{i}(f)\left(u_{j} \cdots u_{1}\right)-\left(u_{j+1} \cdots u_{1}\right)^{*} \varphi_{j+1}(f)\left(u_{j+1} \cdots u_{1}\right)\right\| \\
= & \left\|\varphi_{j}(f)-u_{j+1}^{*} \varphi_{j+1}(f) u_{j+1}\right\|<\varepsilon / 4 .
\end{aligned}
$$

Replacing each homomorphism $\varphi_{j}$ by $\operatorname{Ad}\left(v_{j}\right) \circ \varphi_{j}$ for $j=1, \ldots, l$, and still denoting it by $\varphi_{j}$, one has

$$
\left\|\varphi_{j}(f)-\varphi_{j+1}(f)\right\|<\varepsilon / 4, \quad f \in \mathcal{F} \cdot \mathcal{F}, 0 \leq j \leq l-1 .
$$

Define a unital completely positive linear map $\phi: A \rightarrow C_{1}$ by

$$
\phi(f)(t):=\frac{t_{j+1}-t}{t_{j+1}-t_{j}} \varphi_{j}(f)+\frac{t-t_{j}}{t_{j+1}-t_{j}} \varphi_{j+1}(f), \quad \text { if } t \in\left[t_{j}, t_{j+1}\right] .
$$

Then, by (2.11), the map $\phi$ is $\mathcal{F}$ - $\varepsilon$-multiplicative. By (2.9) and (2.7), one has

$$
\left\|\phi_{*}(\hat{f})-\gamma(\hat{f})\right\|_{\infty}<\varepsilon / 2, \quad f \in \mathcal{F} \text {. }
$$


Note that $A$ and $C$ have cancellation for projections, and also $\mathrm{K}_{0}^{+}(A)=\mathrm{K}_{0}^{+}(C)=\mathbb{Q}^{+}$(unital identification) and $\operatorname{Aff}(\mathrm{T}(A)) \cong \operatorname{Aff}(\mathrm{T}(C))$. By Theorem 4.4 and Corollary 6.8 of [9] (see also Theorem 2.6 of [5] and Theorem 5.5 of [4], expressed in terms of $\mathrm{W}$ instead of $\mathrm{Cu}$ ), it follows that the Cuntz semigroup of $A$ and the Cuntz semigroup of $C$ are isomorphic. Applied to the canonical unital map $\mathrm{Cu}\left(C_{1}\right) \rightarrow \mathrm{Cu}(C) \cong \mathrm{Cu}(A)$, Theorem 1 of [22] implies that there is a unital homomorphism $\psi: C_{1} \rightarrow A$ giving rise to this map, and in particular such that

$$
\psi_{*}=\Xi^{-1} \circ\left(\iota_{1, \infty}\right)_{*} \text { on } \operatorname{Aff}\left(\mathrm{T}\left(C_{1}\right)\right) .
$$

Since the ideal of $\mathrm{Cu}\left(C_{1}\right)$ killed by the map $\mathrm{Cu}\left(C_{1}\right) \rightarrow \mathrm{Cu}(C) \cong \mathrm{Cu}(A)$ is zero, as the map $C_{1} \rightarrow C$ is an embedding, it follows that the map $C_{1} \rightarrow A$ is also an embedding. By (2.12), (2.13), and (2.3), one then has

$$
\left\|\phi_{*} \circ \psi_{*}(\hat{f})-\hat{f}\right\|_{\infty}<\varepsilon, \quad f \in \mathcal{F}
$$

as desired.

Recall that

Definition $2.11([15],[8])$. Let $\mathcal{S}$ be a class of unital $\mathrm{C}^{*}$-algebras. A $\mathrm{C}^{*}$-algebra $A$ is said to be tracially approximated by the $\mathrm{C}^{*}$-algebras in $\mathcal{S}$, and one writes $A \in \mathrm{TA} \mathcal{S}$, if the following condition holds: For any finite set $\mathcal{F} \subseteq A$, any $\varepsilon>0$, and any non-zero $a \in A^{+}$, there is a non-zero sub-C*-algebra $S \subseteq A$ such that $S \in \mathcal{S}$, and if $p=1_{S}$, then

(1) $\|p f-f p\|<\varepsilon, f \in \mathcal{F}$,

(2) $p f p \in_{\varepsilon} S, f \in \mathcal{F}$, and

(3) $1-p$ is Murray-von Neumann equivalent to a subprojection of $\overline{a A a}$.

Denote by $\mathcal{I}$ the class of interval algebras, i.e.,

$$
\mathcal{I}=\left\{\mathrm{C}([0,1]) \otimes F: F \text { is a finite dimensional } \mathrm{C}^{*} \text {-algebra }\right\} .
$$

TAI , then, is the class of $\mathrm{C}^{*}$-algebras which can be tracially approximated by interval algebras.

For TAI algebras, based on Winter's deformation technique ([25] and [18]) and on [19], one has the following classification theorem.

Theorem 2.12 (Corollary 11.9 of [17]). Let $A, B$ be unital separable amenable simple $C^{*}$-algebras satisfying the UCT. Assume that $A, B$ are Jiang-Su stable, and assume that $A \otimes Q \in \mathrm{TAI}$ and $B \otimes Q \in \mathrm{TA \mathcal {I }}$. Then $A \cong B$ if and only if $\operatorname{Ell}(A) \cong \operatorname{Ell}(B)$.

The following is the main result of this note, which asserts that certain abstract $\mathrm{C}^{*}$-algebras are covered by the classification theorem above.

Theorem 2.13. Let $A$ be a separable simple unital $C^{*}$-algebra satisfying the UCT. Assume that $A \otimes Q$ has finite nuclear dimension, $\mathrm{T}(A)=\mathrm{T}_{\mathrm{qd}}(A)$, and $\mathrm{K}_{0}(A) \otimes \mathbb{Q}=\mathbb{Q}$ (identification of order-unit groups). Then $A \otimes Q \in \mathrm{TAI}$.

Proof. This follows from Theorem 2.10] above and Theorem 2.2 of [24] directly. 
Proof of Theorem 1.1. By Proposition 8.5 of [2], as $A \otimes Q$ has finite decomposition rank, $\mathrm{T}(A \otimes$ $Q)=\mathrm{T}_{\mathrm{qd}}(A \otimes Q)$. Furthermore, by [13], $A \otimes Q$ is stably finite and nuclear and so by [1] and [11], $\mathrm{T}(A) \neq \varnothing$. Then $\mathrm{K}_{0}(A \otimes Q)=\mathbb{Q}$ (as order-unit groups), and the statement follows from Theorem 2.13. (The classifiability of $A \otimes \mathcal{Z}$ holds by Theorem 2.12.)

\section{REFERENCES}

[1] B. Blackadar and M. Rørdam. Extending states on preordered semigroups and existence of the quasitrace on $\mathrm{C}^{*}$-algebras. J. Algebra, 152(1):240-247, 1992.

[2] J. Bosa, N. P. Brown, Y. Sato, A. Tikuisis, S. White, and W. Winter. Covering dimension of C*-algebras and 2-coloured classification. 06 2015. URL: http://arxiv.org/abs/1506.03974, arXiv:1506.03974,

[3] N. P. Brown. Invariant means and finite representation theory of $\mathrm{C}^{*}$-algebras. Mem. Amer. Math. Soc., 184(865):viii+105, 2006. URL: http://dx.doi.org/10.1090/memo/0865, doi:10.1090/memo/0865.

[4] N. P. Brown, F. Perera, and A. S. Toms. The Cuntz semigroup, the Elliott conjecture, and dimension functions on $C^{*}$-algebras. J. Reine Angew. Math., 621:191-211, 2008. URL: http://dx.doi.org/10.1515/CRELLE.2008.062, doi:10.1515/CRELLE.2008.062.

[5] N. P. Brown and A. S. Toms. Three applications of the Cuntz semigroup. Int. Math. Res. Not. IMRN, (19):Art. ID rnm068, 14, 2007.

[6] M. Dădărlat and S. Eilers. On the classification of nuclear $\mathrm{C}^{*}$-algebras. Proc. London Math. Soc. (3), 85(1):168-210, 2002. URL: http://dx.doi.org/10.1112/S0024611502013679, doi:10.1112/S0024611502013679

[7] G. A. Elliott, G. Gong, H. Lin, and Z. Niu. The classification of simple separable unital locally ASH-algebras. 06 2015. URL: http://arxiv.org/abs/1506.02308, arXiv:1506.02308,

[8] G. A. Elliott and Z. Niu. On tracial approximation. J. Funct. Anal., 254(2):396-440, 2008. URL: http://dx.doi.org/10.1016/j.jfa.2007.08.005, doi:10.1016/j.jfa.2007.08.005

[9] G. A. Elliott, L. Robert, and L. Santiago. The cone of lower semicontinuous traces on a $\mathrm{C}^{*}$-algebra. Amer. J. Math, 133(4):969-1005, 2011.

[10] G. Gong, H. Lin, and Z. Niu. Classification of finite simple amenable $\mathcal{Z}$-stable $\mathrm{C}^{*}$-algebras. 01 2015. URL: http://arxiv.org/abs/1501.00135, arXiv:1501.00135.

[11] U. Haagerup. Quasitraces on exact $\mathrm{C}^{*}$-algebras are traces. C. R. Math. Acad. Sci. Soc. R. Can., 36(2-3):6792, 2014.

[12] X. Jiang and H. Su. On a simple unital projectionless C*-algebra. Amer. J. Math., 121(2):359-413, 1999. URL: http://muse.jhu.edu/journals/american_journal_of_mathematics/v121/121.2jiang.pdf.

[13] E. Kirchberg and W. Winter. Covering dimension and quasidiagonality. Internat. J. Math., 15(1):63-85, 2004. URL: http://dx.doi.org/10.1142/S0129167X04002119, doi:10.1142/S0129167X04002119.

[14] A. J. Lazar and J. Lindenstrauss. Banach spaces whose duals are $L_{1}$ spaces and their representing matrices. Acta Math., 126:165-193, 1971.

[15] H. Lin. Tracially AF C*-algebras. Trans. Amer. Math. Soc., 353(2):693-722, 2001.

[16] H. Lin. Stable approximate unitary equivalence of homomorphisms. J. Operator Theory, 47(2):343-378, 2002.

[17] H. Lin. Asymptotic unitary equivalence and classification of simple amenable $\mathrm{C}^{*}$-algebras. Invent. Math., 183(2):385-450, 2011. URL: http://dx.doi.org/10.1007/s00222-010-0280-9, doi:10.1007/s00222-010-0280-9.

[18] H. Lin. Localizing the Elliott conjecture at strongly self-absorbing $\mathrm{C}^{*}$-algebras, II. J. Reine Angew. Math., 692:233-243, 2014. doi:10.1515/crelle-2012-0182.

[19] H. Lin and Z. Niu. Lifting KK-elements, asymptotic unitary equivalence and classification of simple $\mathrm{C}^{*}$ algebras. Adv. Math., 219(5):1729-1769, 2008. URL: http://dx.doi.org/10.1016/j.aim.2008.07.011. doi:10.1016/j.aim.2008.07.011. 
[20] H. Matui and Y. Sato. Decomposition rank of UHF-absorbing $\mathrm{C}^{*}$-algebras. Duke Math. J., 163(14):26872708, 2014. URL: http://dx.doi.org/10.1215/00127094-2826908, doi:10.1215/00127094-2826908.

[21] M. Riesz. Sur le problème des moments. iii. Ark. F. Mat. Astr. O. Fys, 17(16):1-52, 1923.

[22] L. Robert. Classification of inductive limits of 1-dimensional NCCW complexes. Adv. Math., 231(5):28022836, 2012. URL: http://dx.doi.org/10.1016/j.aim.2012.07.010, doi:10.1016/j.aim.2012.07.010

[23] K. Thomsen. Inductive limits of interval algebras: the tracial state space. Amer. J. Math., 116(3):605-620, 1994. URL: http://dx.doi.org/10.2307/2374993, doi:10.2307/2374993.

[24] W. Winter. Classifying crossed product C*-algebras. 08 2013. URL: http://arxiv.org/abs/1308.5084, arXiv:1308.5084

[25] W. Winter. Localizing the Elliott conjecture at strongly self-absorbing $\mathrm{C}^{*}$-algebras. J. Reine Angew. Math., 692:193-231, 2014. doi:10.1515/crelle-2012-0082.

Department of Mathematics, University of Toronto, Toronto, Ontario, Canada M5S 2E4

E-mail address: elliott@math.toronto.edu

Department of Mathematics, University of Wyoming, Laramie, WY 82071, USA

E-mail address: zniu@uwyo.edu 